\title{
Broadband Equivalent Circuit Model for a Coplanar Waveguide Line Loaded with Split Ring Resonators
}

\author{
Victor Sanz, ${ }^{1}$ Angel Belenguer, ${ }^{1}$ Alejandro L. Borja, ${ }^{1}$ \\ Joaquin Cascon, ${ }^{1}$ Hector Esteban, ${ }^{2}$ and Vicente E. Boria ${ }^{2}$ \\ ${ }^{1}$ Departamento de Ingeniería Eléctrica, Electrónica, Automática y Comunicaciones, Universidad de Castilla-La Mancha, \\ Escuela Politécnica de Cuenca, Campus Universitario, 16071 Cuenca, Spain \\ ${ }^{2}$ Departamento de Comunicaciones, Universidad Politécnica de Valencia, 46022 Valencia, Spain
}

Correspondence should be addressed to Victor Sanz, victor.sanz@uclm.es

Received 17 September 2012; Accepted 16 October 2012

Academic Editor: Eric Lheurette

Copyright (C) 2012 Victor Sanz et al. This is an open access article distributed under the Creative Commons Attribution License, which permits unrestricted use, distribution, and reproduction in any medium, provided the original work is properly cited.

\begin{abstract}
A new equivalent circuit for a coplanar waveguide loaded with split ring resonators is presented. The traditional circuits that model these devices are only able to characterize the left-handed propagation band, and their response is very similar to the real one within a very limited bandwidth. In contrast, this proposed broadband equivalent circuit is able to portray not only the lefthanded propagation band, but also the right-handed one that occurs at higher frequencies. Besides, the response of this kind of basic cells can be adjusted with the proposed circuit model in a bandwidth close to a decade.
\end{abstract}

\section{Introduction}

Many publications, regarding the design of very compact filters based on Coplanar Waveguide (CPW) lines loaded with Split Ring Resonators (SRRs), can be found in scientific literature. Different examples of such filter designs are [15]. These filters are designed based on an equivalent circuit where the value of the different components can be linked in a very direct way to the physical dimensions of the rings and the line [5-7]. Therefore, using this circuit model — that is relatively easy to calculate-, it is possible to design complex filters formed by the concatenation of various basic cells $[8,9]$. This equivalent circuit provides a very good starting point, so that an appropriately configured optimization algorithm can converge to an acceptable solution.

Initially, the equivalent circuit of [5] was able to characterize the upper right-handed bands. However, in a later publication the authors modified their own equivalent circuit [6]. The new proposal provided a more accurate characterization of the coupling and propagation physical phenomena of the real circuit made with CPW and SRRs, but unfortunately, this modified circuit was not able to model the upper right-handed bands. In this paper a modification of the equivalent circuit of [5-7] is proposed. This new equivalent circuit preserves the rigorous description of the physical mechanisms of the real circuit and, at the same time, is able to simultaneously model the left- and right-handed transmission over a bandwidth close to a decade.

The paper is organized as follows. Section 2 discusses the equivalent circuit derivation. Section 3 presents a specific design and the associated results. The performance is also compared with the traditional equivalent circuit. Finally, in Section 4 , the main conclusions of the study are outlined.

\section{Equivalent Circuit}

The basic cell used for the filters of $[1,2,4,8]$ can be seen in Figure 1 and its equivalent circuit in Figure 2.

In order to simplify the circuit, the symmetry along the CPW longitudinal axis has been applied, so in this equivalent circuit, only half cell needs to be portrayed.

Since the electrical size of the cell is quite small, the $\mathrm{CPW}$ line can be modeled using a series inductor and a parallel capacitor. If symmetry had not been applied, these inductance and capacitance values could have been computed simply by multiplying the inductance and capacitance 


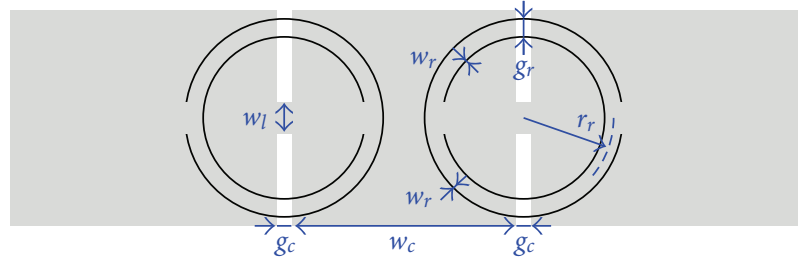

Figure 1: Basic cell geometry. Black is metal on bottom layer; gray is metal on top layer.
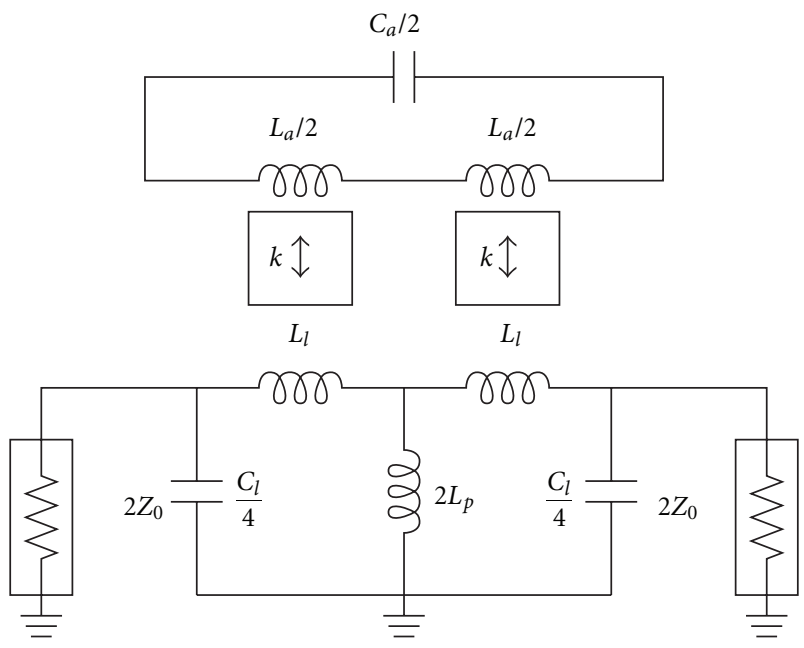

Figure 2: Equivalent circuit.

per unit length of the CPW, $L_{\text {line }}$ and $C_{\text {line }}$, by the line length, $l_{l}$. However, since a symmetry simplification has been considered, the inductance must be multiplied by 2 . Therefore, the line inductor will become $2 L_{l}=2 L_{\text {line }} l_{l}$. Correspondingly, the capacitance must be divided by the same factor. So the line capacitor will be now $C_{l} / 2=C_{\text {line }} l_{l} / 2$.

Furthermore, as can be clearly seen in Figure 1, the CPW is longitudinally divided by a direct connection of the central line and the ground planes. This connection can be modeled with an inductor, $L_{p}$. Since only half a circuit is considered because of the symmetry applied, this inductor is, indeed, equal to $2 L_{p}$.

The central inductor requires that the line be divided into 2 halves. Each half can be modeled with a serial inductor, whose value is $L_{l}=L_{\text {line }} l_{l}$, and a parallel capacitor equal to $C_{l} / 4=C_{\text {line }} l_{l} / 4$.

Finally, it should not be forgotten that the equivalent circuit must be excited with an adapted port. The characteristic port impedance must be equal to $2 Z_{0}$, because the complete coplanar line has been designed with a characteristic impedance equal to $Z_{0}$.

As far as the ring is concerned, it is simply a resonator. Initially [5], it was modeled as an isolated parallel LC tank. But in this model, the inductor is magnetically coupled with the CPW and that coupling excites the ring, and, since the line is broken into two parts by $L_{p}$, the ring must be divided as well. Therefore, the ring inductance is divided into two series-connected inductors, and every half of the ring has to be coupled to the appropriate half of the CPW.

This ring model is valid for an isolated ring [10]. Even other more complicated circuit models like, for example, the model presented in [11], presume an isolated ring as well. However, in the basic cell of Figure 1, one can see that these rings cannot be considered in isolation. The line acts as a ground plane that, in this case, is very close to the rings. This ground plane produces an additional capacitive coupling between the rings. This coupling is clearly different from the direct capacitive coupling which is modeled by $C_{a} / 2$ (see Figure 2).

In order to take this new capacitive coupling into consideration, it is necessary to slightly modify the circuit that models the ring. This modification can be explained from the physical process responsible for the ring resonance.

Within the SRR, first, the current only flows along one of the rings. As this current flows alongside this first ring it progressively crosses to the second one due to their mutual capacitive coupling. When the current reaches the slot in the first ring, it entirely flows along the second ring so that it is not interrupted. From this moment onwards the process inverts itself. The current flows along the second ring and then, due to the capacitive coupling, it is progressively transferred to the first ring. Finally, when the current reaches the second ring slot, it flows, again, entirely along the first ring, just as it did at the beginning of the process.

A circuit that literally models this phenomenon can be seen in Figure 3.

(i) The first ring can simply be replaced by an inductor.

(ii) Then, a series capacitor would replace the capacitive coupling that transfers the current to the second ring.

(iii) Of course the second ring is also modeled by an inductor. Since the second ring is the current destination, it must be connected to the previous capacitor.

(iv) Next, another capacitor can model the capacitive coupling that brings the current back to the first ring.

(v) Finally, the ring inductances are separated into two series inductors in order to perform an appropriate coupling to the line divided by $L_{p}$, just as explained before.

Obviously, this ring model is exactly the same as Figure 2.

However, when the rings are explicitly separated, it is possible to incorporate to the model the capacitive coupling through the line. In order to do that, an additional capacitor, $C_{g}$, connecting the rings, is added to the circuit, as can be seen in Figure 4.

\section{Results}

In order to test the validity of the proposed new equivalent circuit a specific prototype cell has been designed.

This cell has been implemented over a Rogers 4003C substrate of $1.524 \mathrm{~mm}$ thick and designed to show a lefthanded transmission band of around $3 \mathrm{GHz}$ and an input 


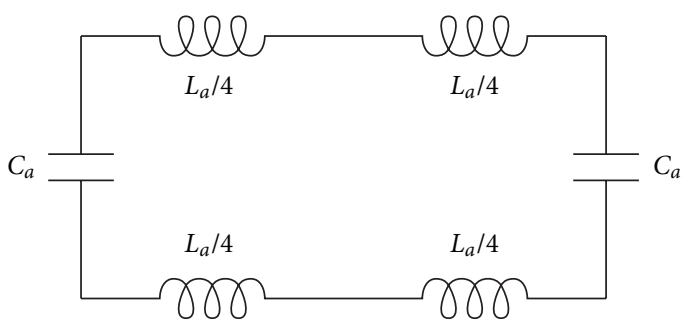

Figure 3: Ring equivalent circuit.
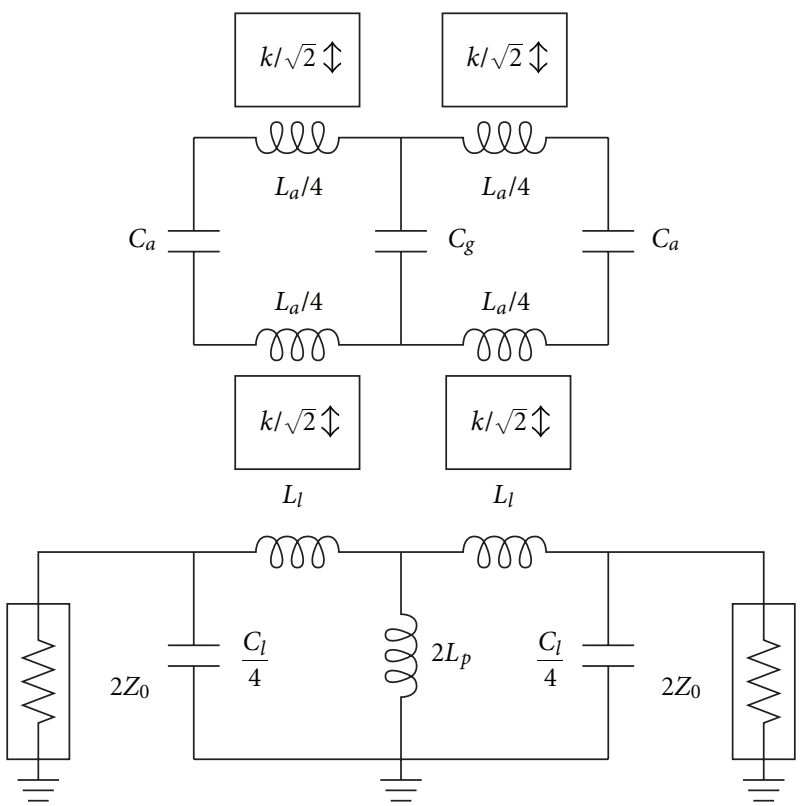

FIgURE 4: Final equivalent circuit.

impedance of $Z_{0}=50 \Omega$. The physical dimensions of the circuit have been calculated by applying the expressions found in [10]. Then, this basic cell has been simulated by using the commercial software Ansoft HFSS and was slightly modified in order to tune the response exactly at $3 \mathrm{GHz}$. These modified dimensions are shown in Table 1.

Similarly, following the recommendations of [10], the components and the coupling coefficient of the original equivalent circuit (Figure 2) have been computed. These values can be seen in the first row of Table 2 .

Finally, the values have been modified by optimization in order to make the HFSS response and the equivalent circuit match around the passband. The optimized values are very similar to the original ones. This fact clearly validates this equivalent circuit.

In Figure 5(a), the HFSS and the equivalent circuit responses are compared and, as mentioned in the introduction, both responses are virtually identical around $3 \mathrm{GHz}$. Unfortunately, the right-handed band that clearly appears in the HFSS response around $7 \mathrm{GHz}$ is completely missing in the equivalent circuit response.

In Figure 5(b) the new equivalent circuit response (Figure 4) and the HFSS-simulated response are compared. The results, at this stage, are clearly better than the results

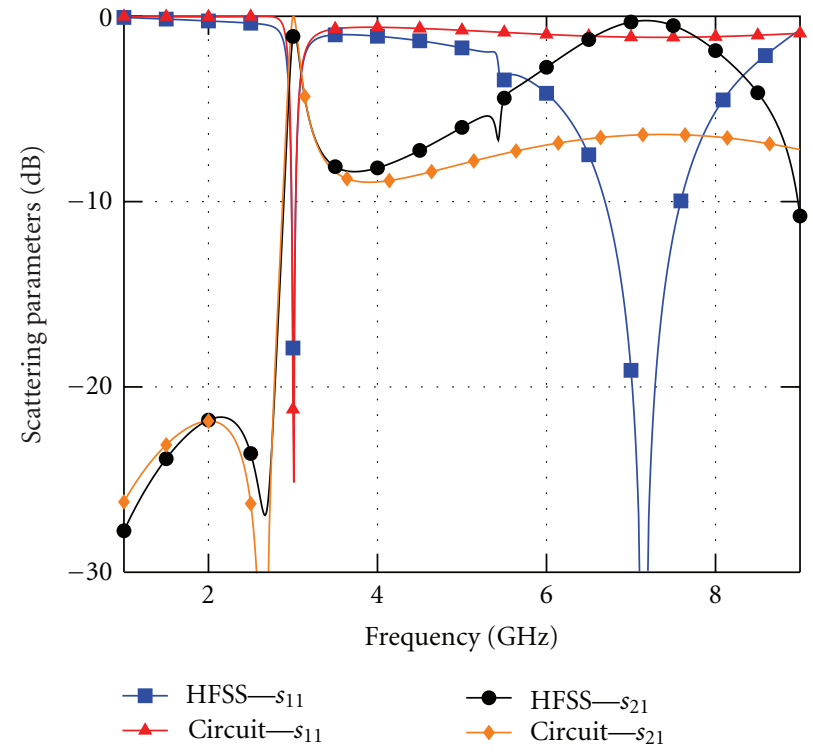

(a)

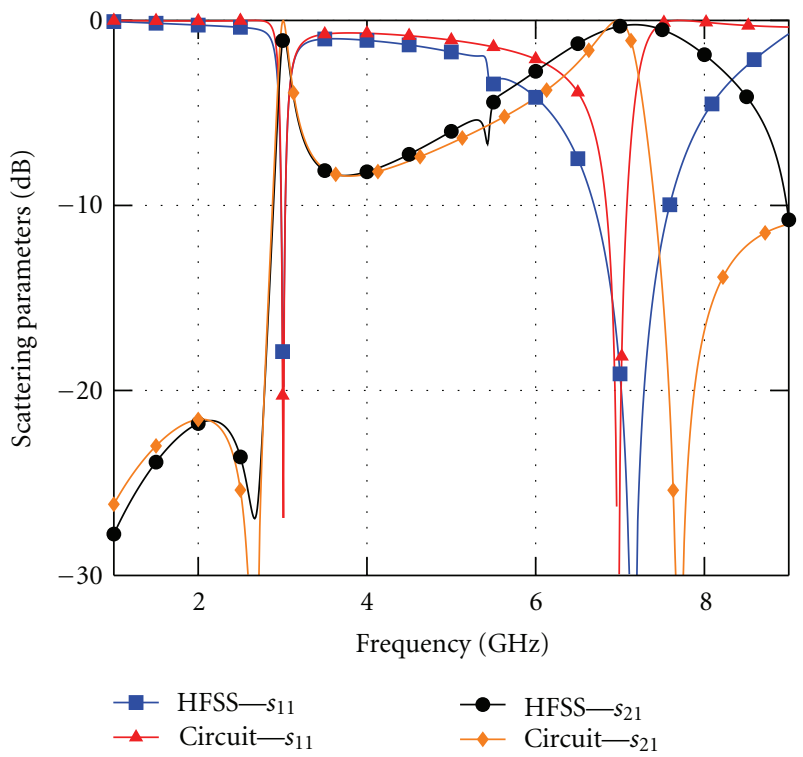

(b)

FIGURE 5: (a) Original equivalent circuit (Figure 2) versus HFSS and (b) equivalent circuit without considering frequency dependence versus HFSS.

of the original equivalent circuit (compare Figure 5(b) with Figure 5(a)).

In fact, the response of this new model and the real response are almost identical under $6 \mathrm{GHz}$. Unfortunately, the equivalent circuit response progressively degrades, as the frequency is increased. Even so, the right-handed band, originally missing, now clearly manifests itself. The aforementioned progressive discrepancy is due to the fact that the dispersive behavior of the CPW line has been completely ignored. Therefore, it is necessary to consider the frequency dependence of the CPW per-unit-length inductance and capacitance if a more accurate response is desired. On 
TABLE 1: Basic cell dimensions.

\begin{tabular}{lccccc}
\hline$w_{c}(\mathrm{~mm})$ & $g_{c}(\mathrm{~mm})$ & $r_{r}(\mathrm{~mm})$ & $w_{r}(\mathrm{~mm})$ & $g_{r}(\mathrm{~mm})$ & $w_{l}(\mathrm{~mm})$ \\
\hline 10 & 0.64 & 3.83 & 0.4 & 0.4 & 1 \\
\hline
\end{tabular}

TABLE 2: Equivalent circuit elements.

\begin{tabular}{lcccccc}
\hline$L_{l}(\mathrm{nH})$ & $C_{l}(\mathrm{pF})$ & $l_{l}$ & $L_{p}(\mathrm{nH})$ & $L_{a}(\mathrm{nH})$ & $C_{a}(\mathrm{pF})$ & 0.299 \\
\hline 1.793 & 0.65 & $2 r_{r}$ & 0.2 & 18.45 & 0.271 \\
1.793 & 0.65 & $2 r_{r}$ & 0.2 & 18.96 & 0.299 & 0.246 \\
\hline
\end{tabular}

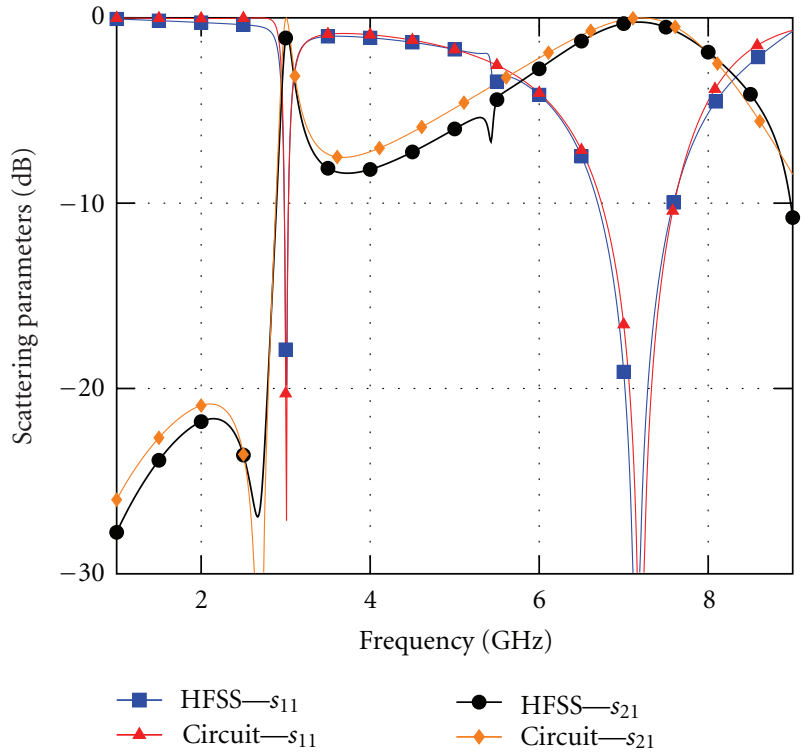

(a)

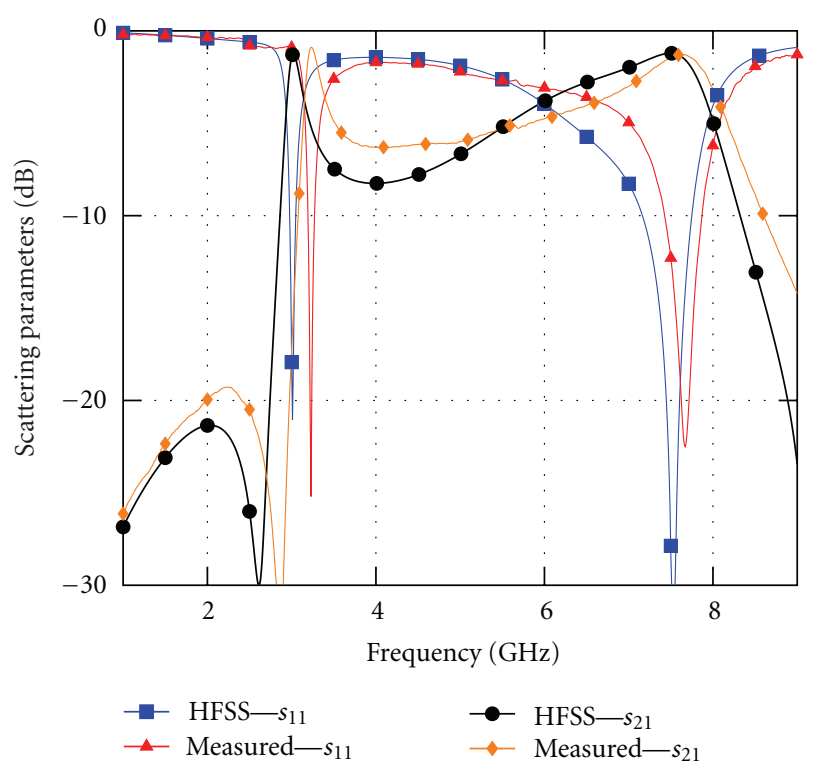

(b)

FIGURE 6: (a) Optimized equivalent circuit (Figure 4) versus HFSS, (b) Results versus HFSS.

the other hand, since the coupling factor depends on the per-unit-length inductance of the line [10], this makes it frequency dependent too.

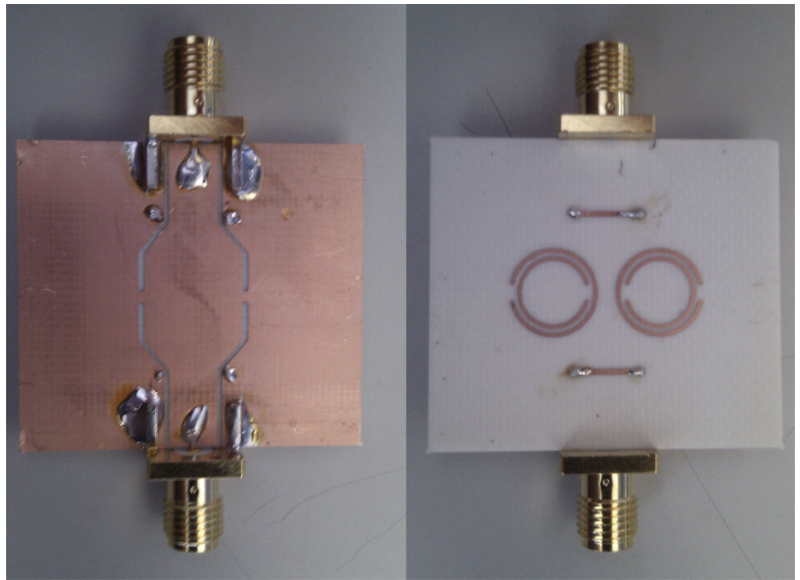

FIGURE 7: Fabricated prototype.

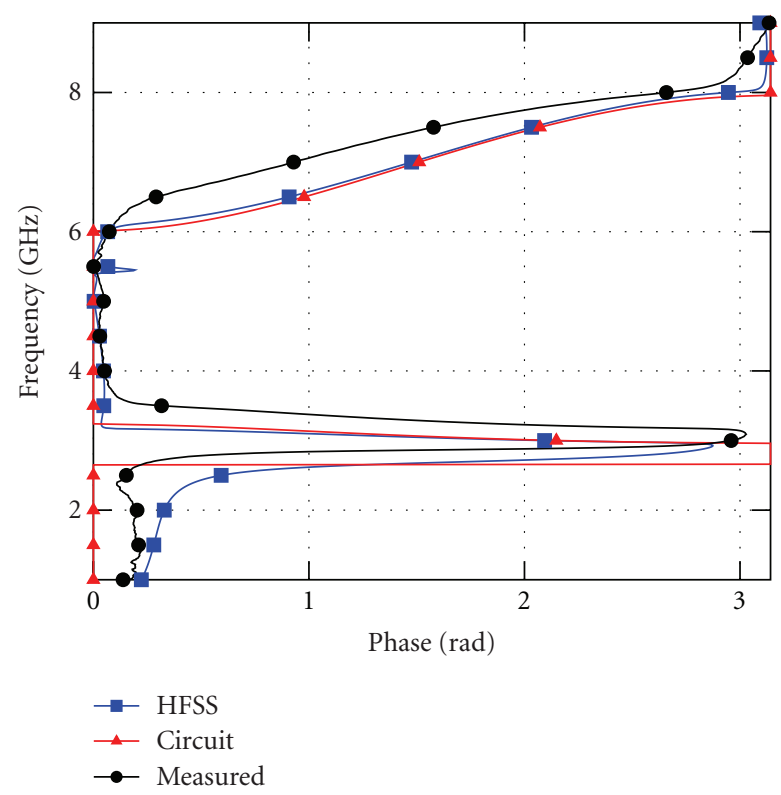

FIgURE 8: Dispersion diagrams.

To achieve a better characterization of the actual behavior of this kind of cells, it has been decided to take into account the frequency dependency of these parameters. To simplify the characterization of the above-mentioned frequency dependency, it has been assumed that it is basically linear. Therefore, to fully characterize this frequency dependencies 
TABLE 3: Frequency dependency of some elements of circuit of Figure 4.

\begin{tabular}{lccc}
\hline & $3 \mathrm{GHz}$ & Slope & Optimized slope \\
\hline$L_{l}$ & $1.793 \mathrm{nH}$ & $0.0531 \mathrm{nH} / \mathrm{GHz}$ & $0.115 \mathrm{nH} / \mathrm{GHz}$ \\
$C_{l}$ & $0.65 \mathrm{pF}$ & $-0.003 \mathrm{pF} / \mathrm{GHz}$ & $-0.003 \mathrm{pF} / \mathrm{GHz}$ \\
$k$ & 0.246 & $0.0039 \mathrm{GHz}^{-1}$ & $0.033 \mathrm{GHz}^{-1}$ \\
\hline
\end{tabular}

it is sufficient to establish the value of these parameters for a specific frequency, in this case $3 \mathrm{GHz}$, and determine the slope.

These parameters have been estimated by using the HFSS port solver. The resulting values are reflected in Table 3 (columns " $3 \mathrm{GHz}$ " and "slope").

The capacitor modeling the capacitive coupling of the rings through the $\mathrm{CPW}, C_{g}$, has been considered a design parameter. By performing a parametric analysis, an initial value for this capacitor has been computed. Specifically $C_{g}=$ $0.125 \mathrm{pF}$ has been selected as the starting value.

Once the initial values have been calculated, the response of the circuit has been optimized. In this case only the slope of the frequency dependences of for the different parameters and $C_{g}$ has been optimized. The rest of values are identical to those obtained after optimizing the original equivalent circuit (Table 2). The optimized slopes can be seen in the last column of Table 3 and, finally, $C_{g}=0.14 \mathrm{pF}$.

The results obtained with this new equivalent circuit are quite similar to the HFSS results (see Figure 6(a)). This fact validates the proposed broadband equivalent circuit.

Finally, a prototype of this cell has been built. In order to be able to measure the cell, a taper and a connector have been added to the input and output ports. A photograph of this prototype (top and bottom) can be seen in Figure 7.

Next, in Figure 6(a) a comparison between the scattering parameters given by HFSS and the measured ones is shown.

In the figure, it can be seen how the experimental results are slightly displaced upwards in frequency. This is basically a consequence of the fabrication process. The prototype has been made using a milling machine. It is impossible to adjust the milling depth with total accuracy. Therefore, prototypes are always slightly overmilled. The main consequence of this overmilling is this upward frequency displacement that can be noticed in the measurements.

Finally, in Figure 8 one can see a comparison of the dispersion diagrams of the equivalent circuit, HFSS results, and experimental data. The dispersion diagrams have been calculated from (1) as explained in [12]:

$$
\beta l_{l}=\cos ^{-1}\left(\frac{1-s_{11} s_{22}+s_{12} s_{21}}{2 s_{21}}\right) .
$$

If the frequency displacement of HFSS results is not taken into account, one can say that the dispersion diagrams really resemble each other. It can be noticed that a lefthanded transmission band appears around $3 \mathrm{GHz}$ and a right-handed transmission band around $7 \mathrm{GHz}$.

\section{Conclusions}

The traditional equivalent circuit that models an SRRloaded CPW has been accordingly modified in order to take into account the additional capacitive coupling that happens through the CPW. When this additional coupling is considered, the circuit is able to characterize not only the left-handed propagation throughout the cell, but also the right-handed propagation. This right-handed band could not be modeled with the previous equivalent circuit. If the right-handed propagation can be modeled by the equivalent circuit, the design of balanced cells with this structure will be notably easier. Wider bandwidths will thus be achieved, and the application range of these devices will be improved.

\section{Acknowledgments}

This work was supported by the Ministerio de Ciencia e Innovación, Spanish Goverment, under Research Projects TEC2010-21520-C04-03 and -01, and by the Autonomous Government of Castilla-La Mancha under Research Projects PPII10-0047-0220 and PPII10-0027-1277.

\section{References}

[1] J. D. Baena, J. Bonache, F. Martín et al., "Equivalent-circuit models for split-ring resonators and complementary splitring resonators coupled to planar transmission lines," IEEE Transactions on Microwave Theory and Techniques, vol. 53, no. 4, pp. 1451-1460, 2005.

[2] A. L. Borja, J. Carbonell, V. E. Boria, and D. Lippens, "Symmetrical frequency response in a split ring resonator based transmission line," Applied Physics Letters, vol. 93, no. 20, Article ID 203505, 2008.

[3] A. L. Borja, A. Belenguer, J. Cascon, H. Esteban, and V. E. Boria, "Wide- band passband transmission line based on metamaterial-inspired CPW bal- anced cells," IEEE Antennas and Wireless Propagation Letters, vol. 10, no. 12, pp. 1421-1424, 2011.

[4] A. L. Borja, J. Carbonell, V. E. Boria, and D. Lippens, "Highly selective left-handed transmission line loaded with split ring resonators and wires," Applied Physics Letters, vol. 94, no. 14, Article ID 143503, 2009.

[5] F. Martín, J. Bonache, F. Falcone, M. Sorolla, and R. Marqués, "Split ring resonator-based left-handed coplanar waveguide," Applied Physics Letters, vol. 83, no. 22, pp. 4652-4654, 2003.

[6] F. Aznar, J. Bonache, and F. Martín, "Improved circuit model for left-handed lines loaded with split ring resonators," Applied Physics Letters, vol. 92, no. 4, Article ID 043512, 2008.

[7] L. J. Roglá, J. Carbonell, and V. E. Boria, "Study of equivalent circuits for open-ring and split-ring resonators in coplanar waveguide technology," IET Microwaves, Antennas and Propagation, vol. 1, no. 1, pp. 170-176, 2007. 
[8] A. L. Borja, J. Carbonell, V. E. Boria, J. Cascon, and D. Lippens, "A 2\% bandwidth C-band filter using cascaded split ring resonators," IEEE Antennas and Wireless Propagation Letters, vol. 9, pp. 256-259, 2010.

[9] M. Gil, J. Bonache, J. García-García, J. Martel, and F. Martín, "Composite right/left-handed metamaterial transmission lines based on complementary split-rings resonators and their applications to very wideband and compact filter design," IEEE Transactions on Microwave Theory and Techniques, vol. 55, no. 6, pp. 1296-1303, 2007.

[10] R. Marqués, F. Martín, and M. Sorolla, Metamaterials with Negative Parameters: Theory, Design and Microwave Applications, John Wiley \& Sons, 2008.

[11] M. Shamonin, E. Shamonina, V. Kalinin, and L. Solymar, "Resonant frequencies of A split-ring resonator: analytical solutions and numerical simulations," Microwave and Optical Technology Letters, vol. 44, no. 2, pp. 133-136, 2005.

[12] C. Caloz and T. Itoh, Electromagnetic Metamaterials: Transmission Line Theory and Microwave Applications, John Wiley \& Sons, 2005. 

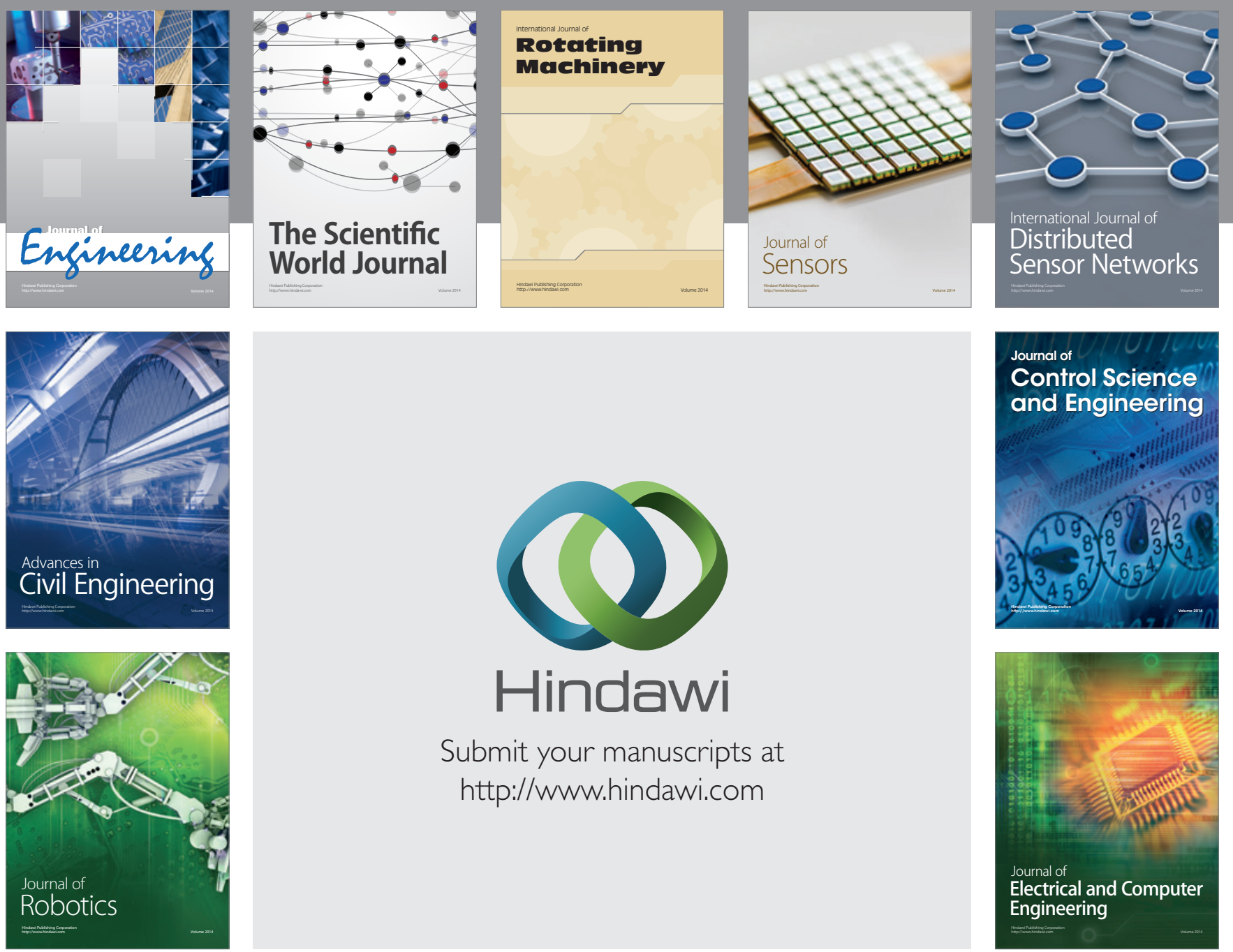

Submit your manuscripts at

http://www.hindawi.com
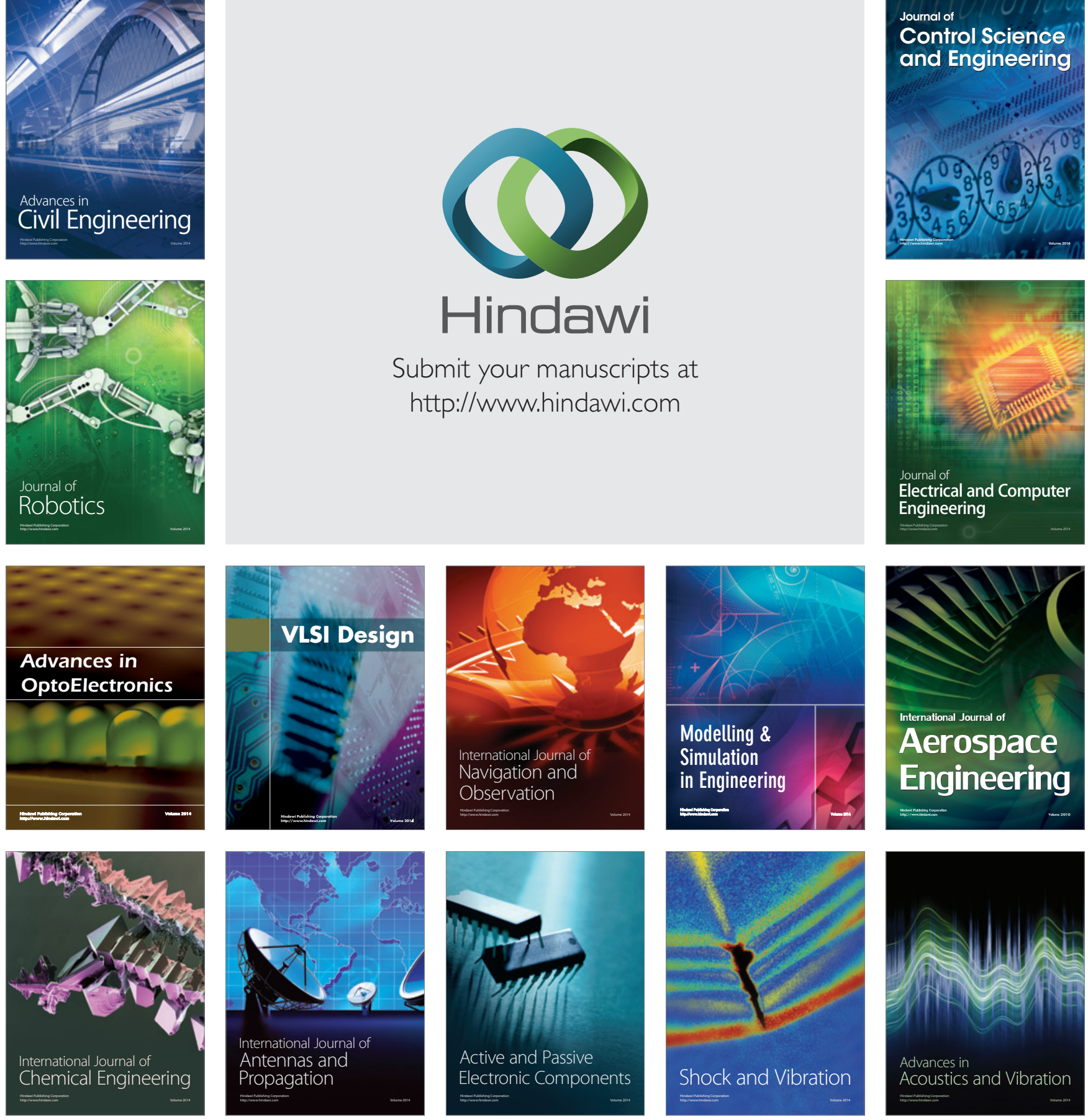